\title{
The Capacitance Modulated by the Convolution between Optical and Electrical Signals Using the GaAsN/GaAs Quantum Well
}

\author{
Jia Feng Wang, Chin Pin Huang, Chen Hao Lin, and Jenn-Fang Chen
}

\begin{abstract}
In this paper, we demonstrate how the alternating photocurrent influences on the impedance of a device. The GaAsN quantum well (QW) is the good device to achieve this purpose. We first show the $\mathrm{QW}$ is sensitive to light and contribute a large photocurrent. And then the QW device receives two signals: a small alternating voltage and a light pulse. The dark current is induced by the applied voltage, and the photocurrent is induced by the light pulse. Both are with the frequency $7 \mathrm{kHz}$. The two transient currents would convolution to each other. As a result of the convolution, a new alternating current with frequency $0.02 \mathrm{~Hz}$ is produced. So the impedance is influenced periodically due to the convoluted current, and the capacitance behaves the sinusoid with frequency $0.02 \mathrm{~Hz}$. We analyze the convolution process, and the experimental data consist with our conclusions. This method is advantageous to the small signals analysis and electrical probing.
\end{abstract}

Index Terms-GaAsN quantum well, impedance, photocurrent.

\section{INTRODUCTION}

The quaternary material (In) GaAsN has recently attracted significant attention, especially with its applications to long-wavelength communication or Quantum Well Infrared Photodetectors (QWIPs) [1]-[4]. In the case of a GaAsN/GaAs QWs, a large band offset can be achieved by depositing a suitable nitrogen composition due to the bandgap bowing effect [5]-[7]. Interactions between carriers confined in the QW and multitudinous defects are observed [8]-[10]. It is easier to generate an electron hole pair in the QW due to the reduced band gap. The carriers will be emitted from the QW with a fast emission time and contribute to the large photo-current (or photo-conduction). Several groups have reported on the fast speed and high frequency characteristics of QWIPs, with response times below 100 ps. [11] The transient photo response of QWIPs is improved in comparison to the bulk, which do not have any band offset structure. In addition to the QWIPs, using the sensitivity of the photo-current generated by the QW, a QW can be a good tool to analyze coupling, interaction, and influences between several small signals. Furthermore, a limited number of studies investigated the convolution between small electrical and optical signals. The purpose of this paper is to analyze the influence of convolution on the impedance.

The capacitance and conductance of a device are typically

Manuscript received March 1, 2014; revised May 22, 2014.

The authors are with the National Chiao Tung University, Hsinchu, Taiwan 30050, and ROC, Taiwan (e-mail: tono.ep98g@nctu.edu.tw, hcp0107@hotmail.com,

jfchen@cc.nctu.edu.tw). probed using a small signal analysis method. In general, a small alternating voltage is applied to the sample and the current is produced with a delayed phase. The impedance can be obtained using the phase delay. The current contains two components: conductive in the real part, and a capacitive current in quadrature in the imaginary part. These two components will couple to each other, and compose a small transient current in response to the small applied alternating voltage [12]. However, if a light pulse is applied to the device, a transient photo-current will be induced. The additional photo-current will also have two components: a conductive and capacitive current, which will also couple with the transient current induced by the small alternating voltage. The process becomes complicated when the light pulse illuminates the device. In order to simplify the scenario, we chose a QW sample with a large conductive current yet small capacitive current. In this case we only have to analyze the optically induced conductive current convolution with the transient current induced by the electrical source. We will begin with the impedance method, since the impedance of the $\omega$-function can be Fourier transformed from the transient current of a $t$-function [12].

\section{SAMPLE DEPOSITION AND FABRICATION}

The GaAsN/GaAs single quantum well (SQW) sample measured in this study was grown using molecular beam epitaxy (MBE) on a ${ }^{+}$-GaAs (001) substrate, with a $0.3 \mu \mathrm{m}$ Si-doped GaAs $\left(4 \times 10^{16} \mathrm{~cm}^{-3}\right)$ buffer layer grown at $580^{\circ} \mathrm{C}$. A $80 \AA$ thick GaAsN layer was then grown at $480{ }^{\circ} \mathrm{C}$, and was undoped in order to avoid the introduction of any unwanted shallow impurities. Finally, a capping layer of $0.3 \mu \mathrm{m}$ Si-doped GaAs $\left(4 \times 10^{16} \mathrm{~cm}^{-3}\right)$ grown at $580{ }^{\circ} \mathrm{C}$ was deposited to terminate the growth. The sample was treated using rapid thermal annealing (RTA) at $800{ }^{\circ} \mathrm{C}$ for 3 minutes to improve the quality of the QW. The photoluminescence (PL) spectrum for as grown QW (non-annealing) is not shown, but we can observe the quality of the $\mathrm{QW}$ and the localized state is improved after annealing. In order to modulate and probe the impedance, the sample is fabricated into a Schottky diode by evaporating Al with a pattern area of $5 \times 10^{-3} \mathrm{~cm}^{-2}$. The PL in this paper was performed using a NAG: $\mathrm{Nd}$ laser at a wavelength of $532 \mathrm{~nm}$, and the admittance spectroscopy was detected using an Agilent HP4194A impedance analyzer. In the impedance measurements, the transient photocurrents are induced using Thorlabs pulsed laser diode with a wavelength of $905 \mathrm{~nm}(1.32 \mathrm{eV})$. The transient electrical currents are induced by the applied alternating voltage with amplitude $0.05 \mathrm{~V}$ in all the measurements of this paper. 
(a)

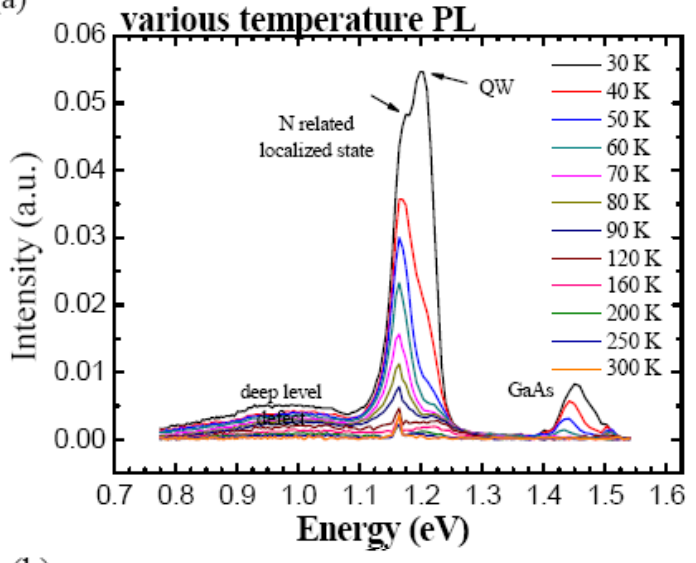

(b)

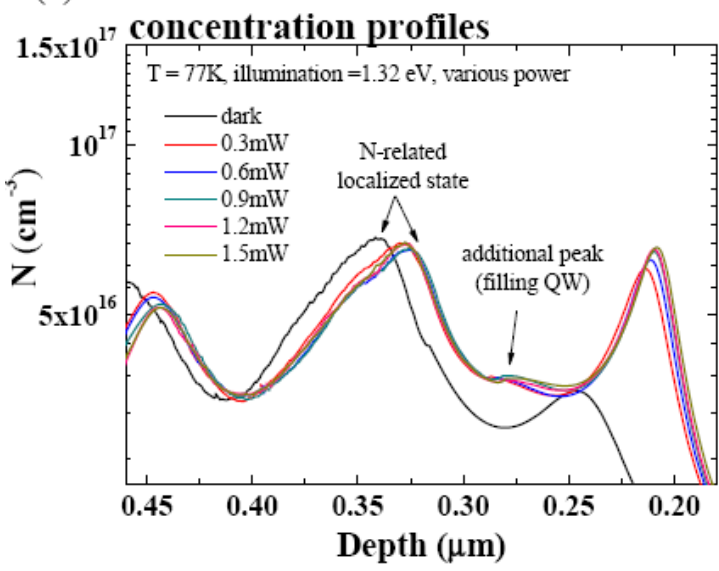

(c)

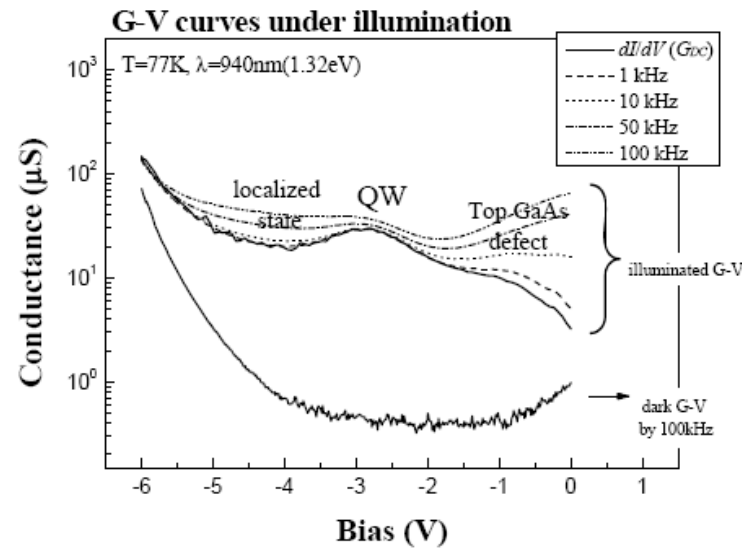

Fig. 1. (Color online) (a) The PL shows the N-related state below the QW level in the energy band. (b) The QW is filled with carriers after illumination. (c) The QW and the localized state contribute a large photo-conductance.

\section{Quantum Well Properties}

We have analyzed the formation and properties of the $\mathrm{N}$-related localized state in the GaAsN/GaAs QW using transmission electron microscopy (TEM) and the deep level transient spectrum (DLTS) [13], [14], which is induced by a nitrogen rich cluster and behaves similarly to the electron level of a quantum dot embedded in the well. The N-related localized state induces a region with a high density of electrons around the QW. The PL spectrum is shown in Fig. 1(a). In the case of the curve at $T=30 \mathrm{~K}$, the peak at $1.20 \mathrm{eV}$ represents the GaAsN QW, and the shoulder at $1.17 \mathrm{eV}$ is the recombination process between the N-related localized state and the valance band of the QW. As the temperature is increased, the $\mathrm{QW}$ peak at $1.20 \mathrm{eV}$ decays more than the peak of the N-related localized state at $1.17 \mathrm{eV}$. This shows that the carriers are easily confined in the localized state at high temperature. The carriers are confined in the QW only at low temperatures $(<50 \mathrm{~K})$, or under strong illumination. Fig. 1(b) shows the concentration profile transformed from the $\mathrm{C}-\mathrm{V}$ curves, the curves are probed in the dark and under illumination at $T=77 \mathrm{~K}$. An additional small peak appears at $0.27 \mu \mathrm{m}$ after illumination, which indicates the carriers are confined in QW under strong illumination. The electron-hole pairs are generated by the light and supply carriers for the QW. The shift in the peaks is caused by a photo-capacitance error in the transformed depth, since the depletion width is proportional to $1 / C$. The frequency-dependent $\mathrm{G}-\mathrm{V}$ curves in Fig. 1(c) are consistent with Fig. 1 (a) and (b). After illumination, both the QW and the localized state contribute to the large photo-conductance, especially the QW due to its faster time constant since it is not related to frequency. In general, the conductance is the summation of the DC current and the charging-discharging current caused by the small alternating current [12].

$$
G(\omega)=G_{D C}+\frac{\omega}{\delta V} \int_{-\infty}^{\infty} \delta I(t) \sin (\omega t) d t
$$

where $G_{D C}$ represents the differential conductance determined by $d I / d V, \delta V$ is the applied small alternating voltage, and $\delta I(t)$ is the responding small alternating current. The AC term in (1) represents the conductive current (real component transformed from the transient current) and is proportional to the AC frequency $\omega$. We have observed the $G_{D C}$ curve is almost a match to the AC conductance at $1 \mathrm{kHz}$ measured by HP4194A. In order to achieve the convolution of the two currents mentioned, we require a sample which responds strongly to light and has no time delay. For this reason the QW structure is a superior design in comparison to the bulk. From the three figures shown in Fig. 1, we have demonstrated that the carriers filling in the QW by the light pulse contribute a large photocurrent with no frequencyresponse. That is, the conditions of the convolution are satisfied.

\section{Convolution Using two Signals}

In this section the QW sample C-t is measured when a light pulse is applied at the same time. We define the angular frequency of the applied small alternating voltage as $\omega_{e}$, and the angular frequency of the pulse light as $\omega_{o}$. If $\omega_{o}$ is close to $\omega_{e}$, a perfect sinusoid is produced in C-t as shown in Fig. 2. After illumination by the light pulse with optical frequency $\omega_{o}$, the admittance, $Y=G_{0}+j \omega C_{0}$, becomes

$$
Y=\left(G_{0}+\Delta G_{0} e^{j \omega_{o} t}\right)+j \omega\left(C_{0}+\Delta C_{0} e^{j \omega_{o} t}\right)
$$

where $\Delta G_{0}$ and $\Delta C_{0}$ are the additional photo-conductance and photo-capacitance respectively, and a time alternating term $\exp \left[-j \omega_{o} t\right]$ is added to describe the light pulse. We can expand (2) as

$$
\begin{aligned}
Y= & \left(G_{0}+\Delta G_{0} \cos \omega_{\mathrm{o}} t-\omega \Delta C_{0} \sin \omega_{o} t\right) \\
& +j \omega\left(C_{0}+\Delta C_{0} \cos \omega_{\mathrm{o}} t+\frac{1}{\omega} \Delta G_{o} \sin \omega_{o} t\right)
\end{aligned}
$$




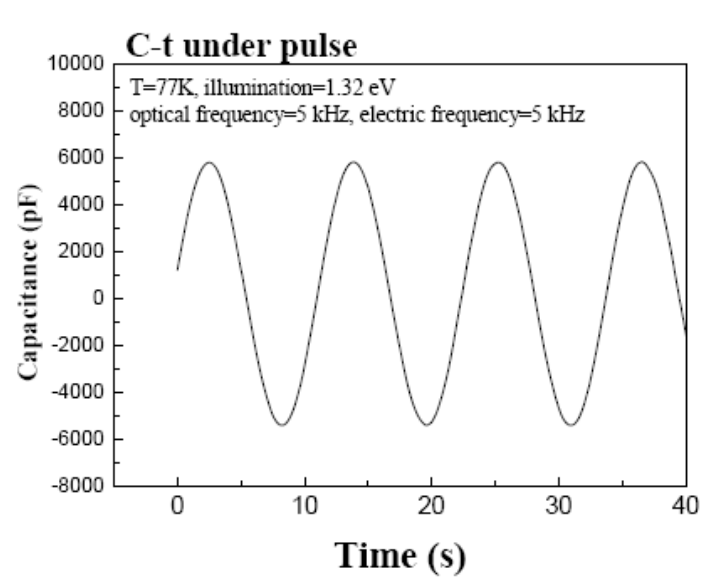

Fig. 2. The unique sinusoidal-waveform displays when $\omega_{o}$ is close to $\omega_{e}$.

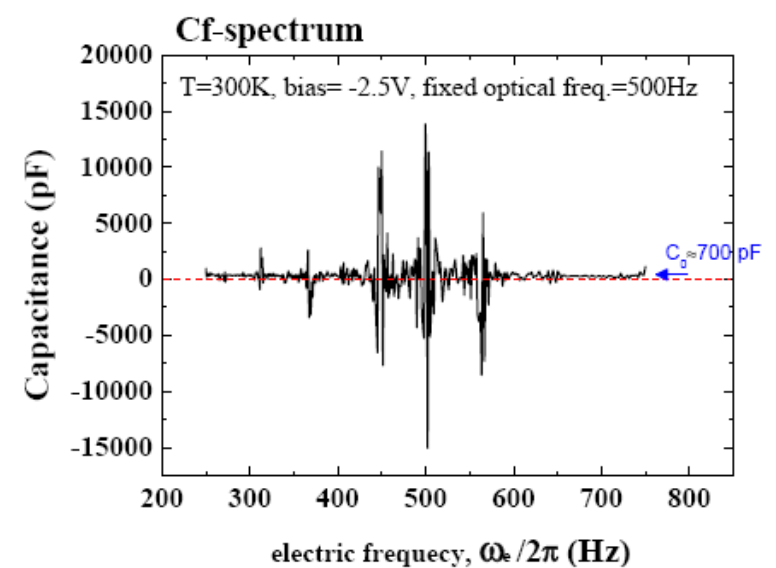

Fig. 3. The capacitance produces a large response only at $\omega_{e} / 2 \pi=500 \mathrm{~Hz}$, and is equal to the normal value $C_{0}$ otherwise. The two minor peaks at 440 and $560 \mathrm{~Hz}$ are likely caused by surrounding interference.

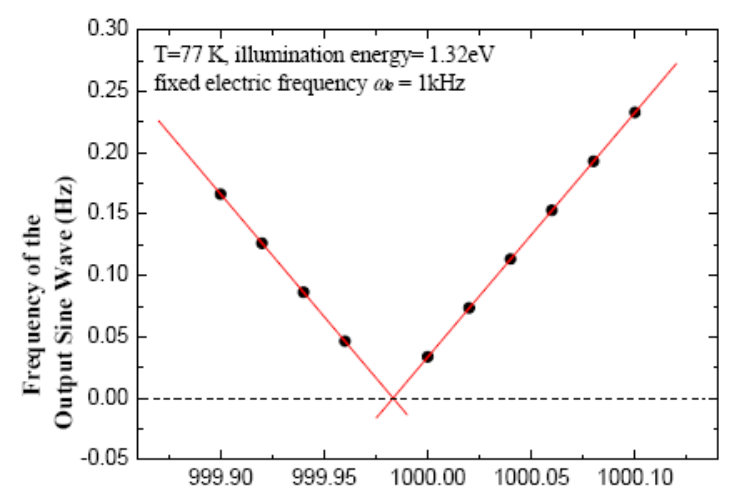

Fig. 4. This figure demonstrates that the frequency of the output sinusoidal-waveform is the difference between $\omega_{o}$ and $\omega_{e}$.

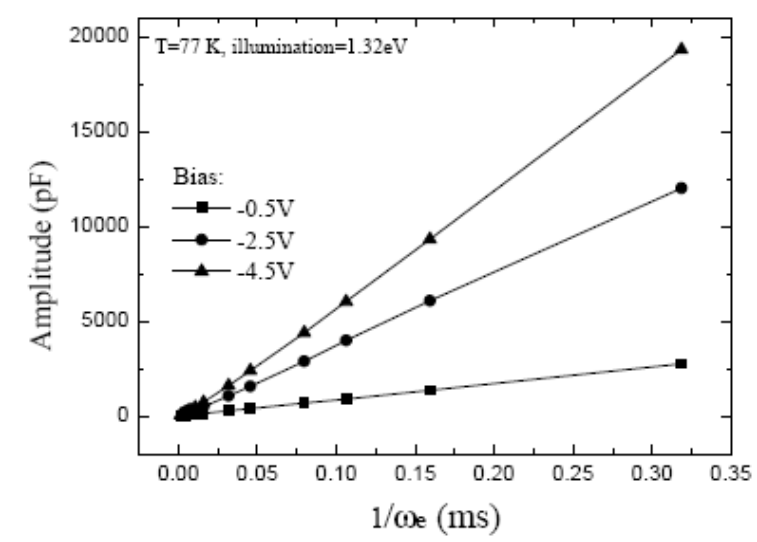

Fig. 5. The amplitudes are proportional to the inverse of $\omega \mathrm{e}$, and the slope is exactly equal to the photo-conductance under the responding bias.
Since the HP4194A impedance analyzer is only able to read the capacitive reactance while measuring C-t, we only consider the imaginary part of (3). For the impedance analyzer, the capacitance can be detected via the convolution of the electrical angular frequency $\omega_{e}$. After the convolution between $\omega_{\mathrm{e}}$ and $\omega_{o}$, the measured capacitance can be obtained from the imaginary term of (3), which can be shown as

$$
\begin{aligned}
C(t)= & C_{0}+\int \Delta C_{0} \cos \omega_{o} t \cos \omega_{e} t d \omega \\
& +\frac{1}{\omega} \int \Delta G_{0} \sin \omega_{o} t \cos \omega_{e} t d \omega
\end{aligned}
$$

According to the principle of an orthogonal function, the integral is equal to 0 if $\omega_{o} \neq \omega_{e}$ and (4) is transformed to

$$
\begin{gathered}
C(t)=C_{0}, \text { if } \omega_{o} \neq \omega_{e}, \\
C(t)=C_{0}+\frac{\Delta C_{0}}{2}\left[\cos \left(\omega_{o}-\omega_{e}\right) t+\cos \left(\omega_{o}+\omega_{e}\right) t\right] \\
+\frac{1}{\omega_{e}} \frac{\Delta G_{0}}{2}\left[\sin \left(\omega_{o}-\omega_{e}\right) t+\sin \left(\omega_{o}+\omega_{e}\right) t\right], \text { if } \omega_{o}=\omega_{e}
\end{gathered}
$$

The term $1 / \omega_{e}$ of (5) also can be written as $1 / \omega_{o}$, it does not matter since $\omega_{o}=\omega_{e}$. Equation (5) can be verified by capacitance-frequency measurements, as shown in Fig. 3.

There is peak at $\omega_{o}=\omega_{e}$, and $C=C_{0}$ otherwise. Since the measurement of $\mathrm{C}$ - $\mathrm{t}$ is limited by the time resolution of the HP4194A, the high frequency term cannot be detected. Equation (5) describes the measured $\mathrm{C}$ - $\mathrm{t}$ value which is similar to the principle of a lock-in amplifier. The measured transient capacitance when $\omega_{o}$ is close to $\omega_{e}$ is

$$
C(t)=C_{0}+\frac{\Delta C_{0}}{2}\left[\cos \left(\omega_{o}-\omega_{e}\right) t\right]+\frac{1}{\omega_{e}} \frac{\Delta G_{0}}{2}\left[\sin \left(\omega_{o}-\omega_{e}\right) t\right]
$$

The second term in (6) can be neglected, since the capacitive current is extremely small in our sample. Equation (6) is dominated by the photo-conductance

$$
C(t)=C_{0}+\frac{1}{\omega_{e}} \frac{\Delta G_{0}}{2}\left[\sin \left(\omega_{o}-\omega_{e}\right) t\right]
$$

We can examine the correctness of (7) by analyzing the frequency and amplitude. In Fig. $4, \omega_{e}$ is fixed at $1 \mathrm{kHz}$ while $\omega_{o}$ is varied. The two straight lines demonstrate the frequency of the sinusoidal oscillation is exactly equal to $\omega_{e}-\omega_{o}$. The more $\omega_{o}$ is varied, the faster the frequency of the oscillation is. The two straight lines cross each other at $f=0$ but $\omega_{0}=999.98$ $\mathrm{Hz}$, indicates a $0.02 \mathrm{~Hz}$ error exists between the electrical source and the light pulse controller. Fig.5 demonstrates that the amplitude of the oscillation is proportional to the reciprocal of the electric frequency $1 / \omega_{e}$. The fitting slope presents the value of the photo-conductance $\left(\sim 10^{-6} \mathrm{~S}\right.$, for example, at $-2.5 \mathrm{~V}$ ) which is comparable to the value measured directly $\left(3.8 \times 10^{-6} \mathrm{~S}\right)$. In Fig. 6, we test the amplitude of the sinusoidal wave at varying bias, and compare them with the photocurrent measured using the HP4194A under a DC light. The point-line chart in Fig. 6 is the photocurrent determined from the convolution method, which is performed at $7 \mathrm{kHz}$. The two components, conductive and capacitive current, of the photocurrent measured by HP4194A are presented by the solid-line and the dash-line in Fig. 6, and both are measured at $1 \mathrm{kHz}$. We can observe the value of the capacitive current, usually written as $\omega C$ [15], is at least one order of magnitude less than the 
conductive current irrespective of the applied bias. The capacitive current is small to be neglected, so the convolution is dominated by the conductive current. These curve trends match, and both have peaks contributed by the QW around -3 $\mathrm{V}$. In addition, the point-line chart is slightly smaller than the solid-line, that is because the photocurrent generated by light pulse is smaller than that generated by DC light.

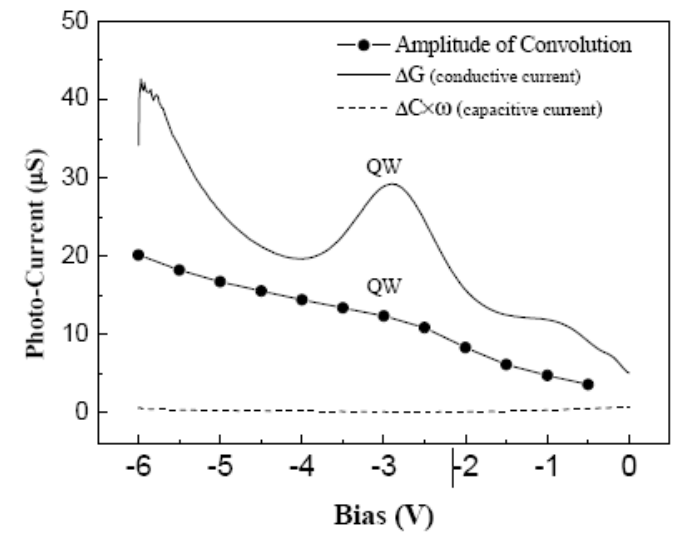

Fig. 6. The solid-line and dash-line are the conductive current and the capacitive current measured by HP4194. The point-line chart is the photo-current determined by convolution method. The capacitive current is small to be neglected, and the convolution is dominated by the conductive current.

In Fig. 4, Fig. 5, and Fig. 6 strong evidence for the validity of (7) is presented. The sinusoidal oscillation arises due to the convolution between the two signals. The conductance as well as the capacitance behaves as a sinusoid with a quadrature delay. We can obtain the function $G(t)$ using the same method of analysis on the real part in (3). We can then give a simple physical explain for the equation obtained in (7). Two currents, one with angular frequency $\omega_{e}$ and the other $\omega_{o}$, are sent to the device. After the superimposition of the two currents, a current with angular frequency $\omega_{o}-\omega_{e}$ is produced. The impedance is influenced by the periodicity $\omega_{o}-\omega_{e}$ and responds to the measured capacitance and conductance.

\section{CONCLUSION}

This phenomenon can be applied on several electrical measurements and small-signals detection. A signal with extremely fast time resolution and small intensity, which is difficult to detect, can be transformed into a signal with a large amplitude (above $\left.10^{3} \mathrm{pF}\right)$ and slow frequency $\left(\omega_{o}-\omega_{e} \sim\right.$ $0.02 \mathrm{~Hz}$ ), which is easier to measure. A complicated and expensive lock-in amplitude system is not required to achieve this function. A device with light responsive conductance, such as a GaAsN/GaAs QW is sufficient. The method presented in this paper is simple and convenient. Furthermore, the $\omega_{o}$ and $\omega_{e}$ values used are in response to the time constant of the photo-current generated by the light. The time resolution of this method can reach under $1 \mathrm{MHz}$, although it is dependent on the illuminating power and the sensitivity of the photo-conductance. However $1 \mathrm{MHz}$ is sufficient for the analysis of most semiconductor devices.

In this study, we first estimated the carrier distribution in a GaAsN/GaAs QW. After illumination, the QW confines a number of carriers and contributes to the large photo-conductance. The photo-conductance produced by the QW can be applied to the analysis of the convolution between several current components, and we have shown how the measured capacitance of a semiconductor device can be modulated by the photo-conductance. This is a powerful technique with applications for small-signal analysis and electrical measurements.

\section{REFERENCES}

[1] I. Vurgaftman, J. R. Meyer, N. Tansu, and L. J. Mawst, "In GaAsN-based type-II "W" quantum-well lasers for emission at $\lambda=1.55 \mu \mathrm{m}$," Appl. Phys. Lett., vol. 83, pp. 2742-2744, Oct. 2003.

[2] W. Li, T. Jouhti, C. S. Peng, J. Konttinen, P. Lakkanen, E. M. Pavelescu, M. Dumitrescu, and M. Pessa, "Low-threshold-current 1.32 $\mu \mathrm{m}$ GaInNAs/GaAs single-quantum-well lasers grown by molecular beam epitaxy," Appl. Phys. Lett., vol. 79, pp. 3386-3388, Nov. 2001.

[3] R. Fehse, S. Tomic, A. R. Adams, S. J. Sweeney, E. P. O’Reilly, A. Andreev, and H. Riechert, "A Quantitative study of radiative, arger, and defect related reconbination processes in 1.3- $\mu \mathrm{m}$ GaInNAs based quantum-well lasers," IEEE J. Sel. Top. Quantum Electron, vol. 8, pp. 801-810, Jul. 2002.

[4] M. Fischer, D. Gollub, M. Reinhardt, M. Kamp, and A. Forchel, "GaInNAs for GaAs based lasers for the 1.3 to $1.5 \mu \mathrm{m}$ range," J. Cryst. Growth, vol. 251, pp. 353-359, Apr. 2003.

[5] L. Vegard, "Die Konstitution der Mischkristalle und die Raumfüllung der Atome," Zeitschrift Für Physik, vol. 5, pp. 17-26, 1921.

[6] I. Vurgaftman, J. R. Meyer, and L. R. R. Mohan, "Band parameters for III-V compound semiconductors and their alloys," J. Appl. Phys. vol. 89 , pp. 5815-5875, Jun. 2001.

[7] M. Weyers and M. Sato, "Growth of GaAsN alloys by low pressure metalorganic chemical vapor deposition using plasma-cracked $\mathrm{NH}_{3}$," Appl. Phys. Lett., vol. 62, pp. 1396-1398, Mar. 1993.

[8] R. Nafarajan, T. Fukushima, S. W. Corzine, and J. E. Bowers, "Effects of carrier transport on high-speed quantum well lasers," Appl. Phys. Lett. vol. 59, pp.1835-1837, Jul. 1991.

[9] N. Tansu and L. J. Mwast, "Current injection efficiency of InGaAsN quantum-well lasers," J. Appl. Phys. vol. 97, pp. 054502-1 -054502-18, Feb. 2005.

[10] Z. Y. Zhao, C. Yi, K. R. Lantz, and A. D. S. Roberts, "Effect of donor-complex-defect-induced diople field on InAs/GaAs quantum dot infrared photodetector activation energy," Appl. Phys. Lett. vol. 90, pp. 233511-1 -233511-3, Jun. 2007

[11] M. R. X. de Barros, P. C. Becker, R. S. Miranda, L. C. West, J. Dunkel, C. W. Robert, J. W. Stayt, and J. V. Swaminathan, "Response time measurement of a mid-infrared photodetector," Electron. Lett, vol. 30, no. 13, pp. 1093-1904, Jun. 1994.

[12] S. E. Laux, "Techniques for small-signal analysis of semiconductor devices," IEEE Trans. Comput.-Aided Des, vol. CAD-4, pp. 472-481, Oct. 1985 .

[13] J. F. Chen, P. C. Hsieh, R. S. Hsiao, J. S. Wang, and J. Y. Chi, "Evidence for the electron trap state associated with N-rich clusters in InGaAsN/GaAs quantum wells," Nanotechnology, vol. 18, pp. 235402-1 -235402-5, May 2007.

[14] J. F. Chen, R. S. Hsiao, P. C. Hsieh, J. S. Wang, and J. Y. Chi, "Effect of growth rate on the composition fulctuation of InGaAsN/GaAs single quantum wells," J. Appl. Phys, vol. 99, pp. 123718-1 -123718-5, Jun. 2006.

[15] G. Vincent, D. Bois, and P. Pinard, "Conductance and capacitance studies in GaP Schottky barriers," J. Appl. Phys, vol. 46, pp. 5173-5178, 1975.

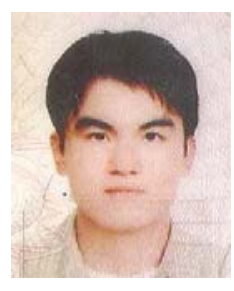

Jia-Feng Wang was born in Kaohsiung, Taiwan in 1984. He received his M. S. degree from National Changhua Education University, Changhua, Taiwan in 2008. He is pursuing $\mathrm{PhD}$ in National Chiao Tung University, Hsinchu, Taiwan. In early period, his research interests are magnetic material and nanotechnology. Now He focuses on the III-V semiconductor material, the quantum dots and the quantum well.

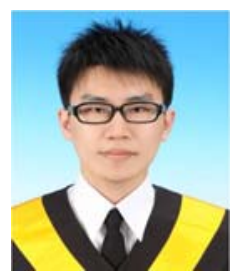

Chin-Pin Huang was born in Hsinchu, Taiwan in 1990. He received his bachelor of engineering in National Kaohsiung University in 2012. He is pursuing M. S. degree in Department Electrophysics, National Chiao Tung University. His research in semiconductor is about electrical and optical analysis of $\mathrm{GaAsN} / \mathrm{GaAs}$ quantum well. 


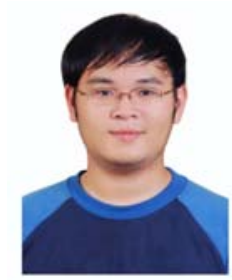

Chen Hao Lin was born in Hsinchu, Taiwan in 1990. He received his bachelor degree from the Department of Applied Physics at National University of Kaohsiung in 2012. And he is now pursuing master's degree in the Department of Electrophysics at National Chiao Tung University in Hsinchu, Taiwan.

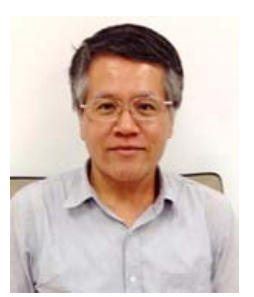

Jenn Fang Chen received his M. S. degree in the Department Electronics Engineering, National Chiao Tung University in 1981. He received his $\mathrm{PhD}$ in the Department of electrical and computer engineering, State University of New York at Buffalo in 1989. During 1985-1989, he was the postductor in AT \& Bell Labs. Now he is the professor in Department Electrophysics, National Chiao Tung University, Hsinchu, Taiwan. His has keen interest in semiconductor material. 